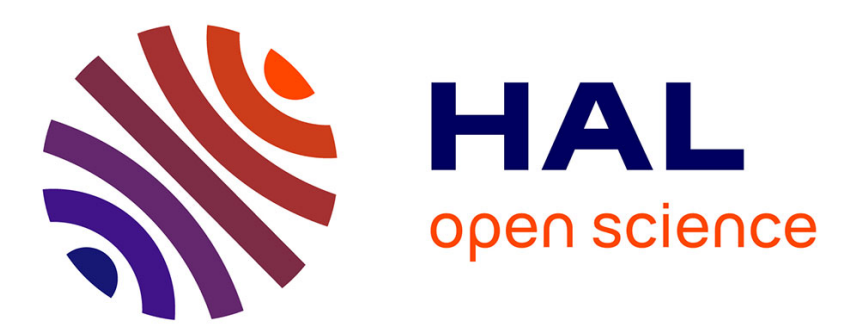

\title{
Incidence de modèles rhéologiques simples sur la simulation d'un ouvrage d'art en service
}

\author{
Yvon Riou, Christophe Nicolas, Nassurdine Abdallah
}

\section{To cite this version:}

Yvon Riou, Christophe Nicolas, Nassurdine Abdallah. Incidence de modèles rhéologiques simples sur la simulation d'un ouvrage d'art en service. Revue Française de Génie Civil , 1998, 2 (6), pp.667-689. 10.1080/12795119.1998.9692199 . hal-01006962

\section{HAL Id: hal-01006962 \\ https://hal.science/hal-01006962}

Submitted on 15 Mar 2017

HAL is a multi-disciplinary open access archive for the deposit and dissemination of scientific research documents, whether they are published or not. The documents may come from teaching and research institutions in France or abroad, or from public or private research centers.
L'archive ouverte pluridisciplinaire HAL, est destinée au dépôt et à la diffusion de documents scientifiques de niveau recherche, publiés ou non, émanant des établissements d'enseignement et de recherche français ou étrangers, des laboratoires publics ou privés. 


\section{Incidence de modèles rhéologiques simples sur la simulation d'un ouvrage d'art en service}

\section{Yvon Riou - Christophe Nicolas - Nassurdine Abdallah}

Laboratoire de Génie Civil de Nantes Saint Nazaire, Ecole Centrale de Nantes, BP 92101, F-44321 Nantes cedex 03

RÉSUMÉ. Ces vingt dernières années ont vu se développer avec la géotechnique " numérique " de nombreux modèles de comportement dont le degré de sophistication et le nombre de paramètres sont sans cesse croissants. L'objectif du travail présenté ci-dessous est de montrer dans quelle mesure deux modèles de comportement, implantés sur un code d'éléments finis, permettent de simuler les déformations d'un sol pulvérulent aux abords d'un tunnel revêtu à faible couverture. Les trois modèles de comportement retenus sont ceux de Mohr-Coulomb, de Vermeer et une adaptation de ce dernier, modèles pour lesquels les moyens à mettre en cuvre lors de l'identification des paramètres sont considérés comme classiques. Pour juger de la pertinence des résultats, les simulations numériques sont confrontées à des mesures effectuées sur un modèle réduit centrifugé représentant la section courante d'un tunnel revêtu soumis à un chargement superficiel.

ABSTRACT. Development of sophisticated models for a better representation of materials behaviour has received considerable attention in the past decades. In order to reproduce foundamental mechanical characteristics of materials, the constitutive relations consider more and more parameters. The purpose of the investigation in this paper is to seek how simple models, elastic perfectly plastic and seven parameters strain hardening models, can explain the bidimensional ground displacements around a shallow tunnel subjected to a monotonic load. The results of two centrifuge model tests are examined to check the numerical approach.

MOTS-CLÉS : modélisation, éléments finis, bidimensionnel, tunnel, centrifugeuse, loi de comportement, comparaison calcul-mesures.

KEYWORDS: modelling, finite element, bidimensional, tunnel, centrifuge, constitutive relations, computation-measurements comparison. 


\section{Avant-propos}

L'objectif du travail présenté ci-dessous est de tester des modèles de comportement dans un calcul d'ouvrage. Comme ouvrage de référence, nous avons choisi une section courante de tunnel à faible couverture dans un terrain meuble. Les raisons qui nous ont amenés à opter pour ce type de configuration viennent de la demande pressante des pouvoirs et entreprises publics confrontés à l'estimation des désordres occasionnés par les interactions fondations - tunnels.

Les membres du projet «Validation des codes de calcul » du GRECO Géomatériaux II avaient, en 1994, procédé à des confrontations modélisation numérique modélisation physique en centrifugeuse. Le déconfinement en section courante d'un tunnel non revêtu avec chargement superficiel avait notamment été étudié en considérant divers modèles de comportement [DAR 95]. L'essentiel des conclusions établies sur les modélisations numériques réalisées « en aveugle » et rapportées dans le bilan du GRECO Géomatériaux II, se résumait de la façon suivante :

- un modèle de Mohr-Coulomb peu approprié à l'estimation des déplacements dans le massif de sol, lors des phases de déconfinement et de chargement,

- un modèle CJS et un modèle de Nova plus réalistes tout particulièrement lors de la phase de déconfinement (modèle CJS) et lors de la phase de chargement (modèle de Nova).

On rappelle ici que, de ces trois modèles, seul le modèle CJS proposait un écrouissage cinématique.

Comme dans l'étude citée précédemment, nous considérons une section courante de tunnel à déformation plane, limitant ainsi le calcul à un problème bidimensionnel. Dans le cas présent, le tunnel est revêtu et le massif de sol est chargé en surface par l'intermédiaire d'une semelle rigide. Dans un premier temps, la modélisation numérique, menée sur le code de calcul par éléments finis CESAR-LCPC, est traitée avec les modèles de Mohr-Coulomb et de Vermeer. Par la suite, nous proposons un modèle intermédiaire, en terme de sophistication, susceptible d'alléger la procédure de résolution du système non linéaire. Une étude paramétrique, permettant de fournir des informations sur l'incidence du modèle lui-même et sur l'incidence du choix des paramètres dans la modélisation de l'ouvrage étudié, complète cette étude. La comparaison des résultats numériques avec les mesures expérimentales porte sur le tassement sous la fondation et sur la déformation horizontale du tunnel.

\section{Configuration étudiée}

Une description complète du dispositif d'essai en centrifugeuse ne se justifie pas ici. Seule une présentation succincte de son principe est fournie ci-dessous. Pour de plus amples renseignements, on se reportera au document de Chambon [CHA 94].

L'essai a été réalisé sur la centrifugeuse du LCPC-Centre de Nantes. Le tunnel est représenté par un tube d'Ertacétal de $100 \mathrm{~mm}$ de long, $100 \mathrm{~mm}$ de diamètre et 
$5 \mathrm{~mm}$ d'épaisseur, disposé dans un container rempli de sable. Ce remplissage est effectué par pluviation suivant l'axe longitudinal du tunnel, i.e. suivant une direction différente de la gravité artificielle. Le poids volumique du matériau est de $15,5 \mathrm{kN} / \mathrm{m}^{3}$. La couverture $\mathrm{C}$ est égale au diamètre $\mathrm{D}$ du tube. Ce rapport $\mathrm{C} / \mathrm{D}$, égal à 1 , justifie ainsi la désignation de tunnel à faible couverture. Le chargement du sol en surface est assuré par une plaque rigide de $40 \mathrm{~mm}$ de largeur placée dans l'axe du tunnel. Une couche de sable, collée sur la paroi externe du tube, donne au contact sol-tunnel un caractère rugueux. Avant d'appliquer le chargement superficiel d'une valeur de $1 \mathrm{MPa}$ sur la fondation, le container et son dispositif expérimental sont soumis à une accélération de $50 \mathrm{~g}$. La configuration ainsi testée en centrifugeuse, représente un massif de sol dont les dimensions "prototype " sont indiquées sur la figure 1. Les dimensions du tube d'Ertacétal sont telles que la rigidité diamétrale est équivalente à celle d'un voussoir prototype en béton d'un diamètre identique mais dont l'épaisseur est de $15 \mathrm{~cm}$. Dans tout ce qui suit, les caractéristiques dimensionnelles et les déplacements font référence à ce prototype.

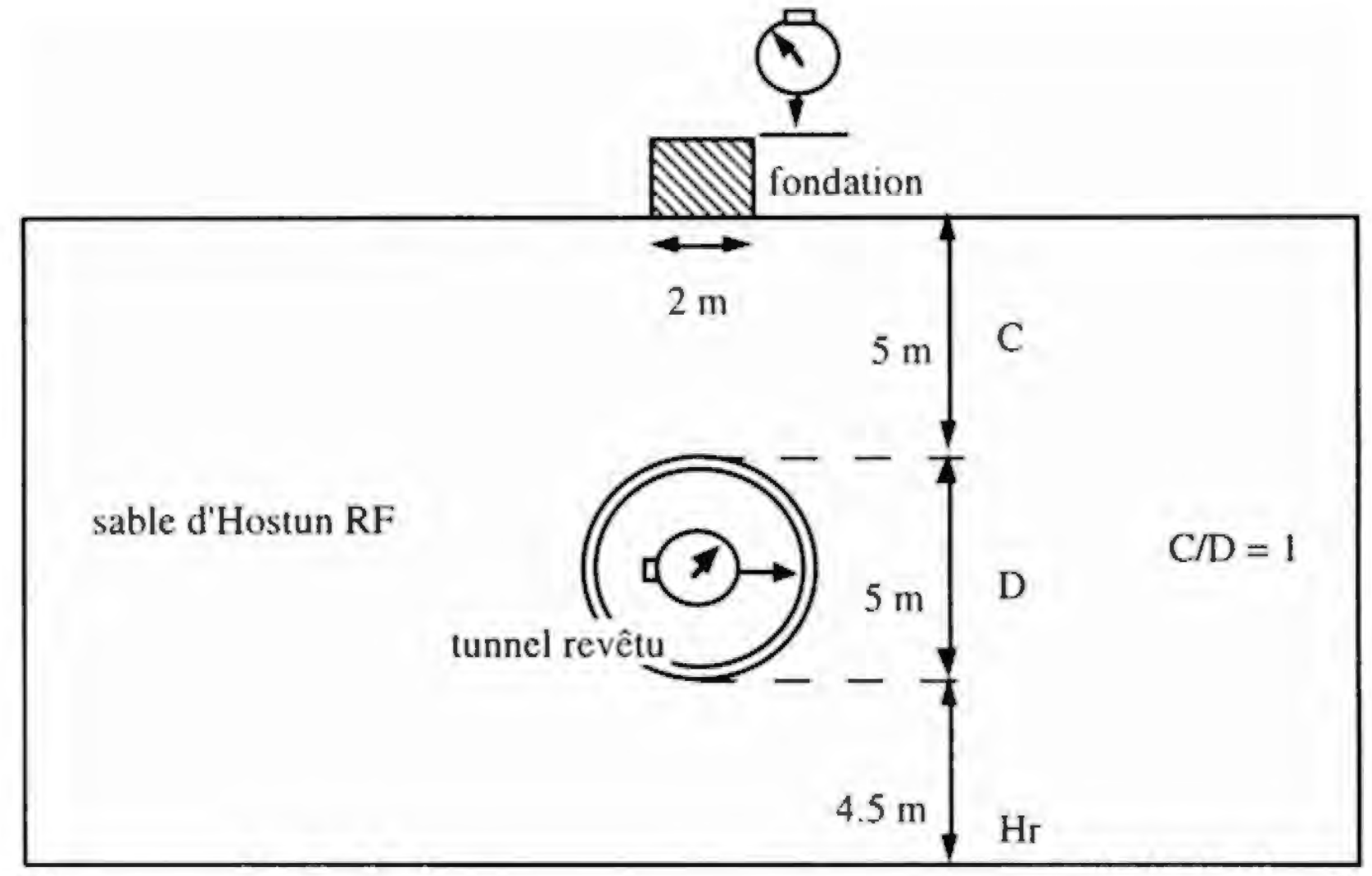

$40 \mathrm{~m}$

Figure 1. Configuration de l'essai en centrifugeuse, dimensions prototype. Schematic of the centrifuge model, prototype dimensions

\section{Modélisation du comportement du sol}

Pour représenter le comportement du sable, nous avons retenu, dans un premier temps, deux modèles à degrés de sophistication différents : le modèle de MohrCoulomb et celui de Vermeer. 


\subsection{Modèle de Mohr-Coulomb}

Le modèle de Mohr-Coulomb est exploité ici dans sa version non associée. Nous considérons que sa formulation est suffisamment connue pour ne pas faire l'objet de commentaires particuliers.

\subsection{Modèle de Vermeer}

Le modèle de Vermeer, spécifique aux matériaux pulvérulents, a été présenté en 1982 [VER 82]. Ce modèle à 5 ou 7 paramètres selon les versions est plus complet que le précédent puisque intégrant un deuxième mécanisme, celui de consolidation, et un écrouissage. Compte tenu de sa très faible diffusion tant dans le domaine industriel qu'universitaire, et des diverses versions dont il a fait l'objet, nous proposons une description rapide de ses principes et de la version exploitée dans cette étude.

\section{Comportement élastique}

Dans sa version originale, le modèle considère une hyperélasticité non linéaire à coefficient de Poisson nul. En raison des temps de calcul parfois prohibitifs et des problèmes de résolution numérique occasionnés par cette non-linéarité, nous avons adopté la version à élasticité linéaire exprimée en termes de module d'Young (E) et coefficient de Poisson (v) constants, proposée par Tadjbakhsh et Frank [TAD 85].

\section{Comportement plastique}

Comme dans toute modélisation se rapportant aux milieux pulvérulents, le mécanisme de cisaillement rend compte du phénomène de contractance dilatance. A la différence du modèle de Mohr-Coulomb, les passages de l'état contractant à l'état dilatant et de l'état élastique à l'état limite s'effectuent de manière continue. Cette propriété est assurée par une fonction de charge et par un potentiel plastique non associé qui dépendent d'un paramètre d'écrouissage.

$$
\begin{aligned}
& F_{c}\left(\sigma, r_{c}\right)=I_{1 \sigma} \cdot I_{2 \sigma}+I_{3 \sigma} \cdot A\left(r_{c}\right)=0 \quad r_{c}=r_{c}\left(\bar{\varepsilon}_{c}^{p}\right): \text { paramètre d'écrouissage } \\
& \mathrm{I}_{1 \sigma}, \mathrm{I}_{2 \sigma}, \mathrm{I}_{3 \sigma}: 3 \text { invariants du tenseur des contraintes, } \\
& \mathrm{A}(\chi)=\frac{27 \cdot(3+\mathrm{H}(\chi))}{(2 \cdot \mathrm{H}(\chi)+3) \cdot(3-\mathrm{H}(\chi))}, \mathrm{H}(\chi)=\sqrt{\left(\frac{\chi}{2}\right)^{2}+\mathrm{c} \cdot \chi}-\frac{\chi}{2} \\
& \chi=\bar{\varepsilon}_{\mathrm{c}}^{\mathrm{p}} \cdot \frac{2 \cdot \mathrm{G}_{0}}{\mathrm{P}_{0}} \cdot\left(\frac{\mathrm{P}_{0}}{\bar{\sigma}}\right)^{\beta}, \bar{\sigma}=\sqrt{\frac{\sigma_{\mathrm{ij}} \sigma_{\mathrm{ij}}}{3}}, \mathrm{G}_{0}=\frac{3 \cdot \mathrm{P}_{0}}{2 \cdot \varepsilon_{0}^{\mathrm{e}}} \\
& c=\frac{6 \cdot \sin \phi_{p}}{3-\sin \phi_{p}} \\
& \mathrm{P}_{0} \quad: \text { Pression isotrope de référence } \\
& \bar{\varepsilon}_{\mathrm{c}}^{\mathrm{p}} \quad: \text { module de déformation plastique déviatorique } \\
& \varepsilon_{0}^{\mathrm{e}} \quad \text { : déformation volumique élastique à la pression } \mathrm{P} 0 \\
& \phi_{\mathrm{p}} \quad \text { : angle de frottementinterne }
\end{aligned}
$$


Ce paramètre est un scalaire et confère à l'écrouissage un caractère isotrope.

Le potentiel plastique de la version originale du modèle présente une forme analogue à celle de la surface de charge. Toutefois, cette formulation nécessite un traitement lourd pour la détermination du sommet de la surface conique. Aussi, nous avons exploité la version à potentiel plastique simplifié proposé par Vermeer :

$$
\begin{array}{rlrl}
\mathrm{G}_{\mathrm{c}}\left(\sigma, \psi_{\mathrm{m}}\right) & =\frac{2}{3} \cdot \mathrm{q}-\frac{4}{3} \cdot \mathrm{p} \cdot \sin \left(\psi_{\mathrm{m}}\right) & \\
\mathrm{q} & =\left(\frac{3}{2} \cdot \mathrm{s}_{\mathrm{ij}} \cdot \mathrm{s}_{\mathrm{ij}}\right)^{0,5} & \mathrm{~s}_{\mathrm{ij}}=\sigma_{\mathrm{ij}}-\mathrm{p} \cdot \delta_{\mathrm{ij}} \\
\text { avec } \quad \mathrm{p}: \text { pression moyenne } & \Psi_{\mathrm{m}}: \text { angle de dilatance } 1
\end{array}
$$

Contrairement au modèle de Mohr-Coulomb, ce mécanisme de cisaillement est complété par un mécanisme de consolidation permettant de représenter les déformations irréversibles lors d'une compression isotrope. Nous avons gardé, dans cette étude, la formulation de base de la surface de charge :

$$
\begin{aligned}
\mathrm{F}_{\mathrm{v}}\left(\sigma, \bar{\varepsilon}_{\mathrm{v}}^{\mathrm{p}}\right)=\varepsilon_{0}^{\mathrm{c}} \cdot\left(\frac{\bar{\sigma}}{\mathrm{P}_{0}}\right)^{\beta}-\bar{\varepsilon}_{\mathrm{v}}^{\mathrm{p}} \\
\quad \varepsilon_{0}^{\mathrm{c}}: \text { déformation volumique plastique à la pression } \mathrm{P} 0, \\
\text { avec } \quad \bar{\varepsilon}_{\mathrm{v}}^{\mathrm{p}}: \text { déformation volumique plastique, paramètre d'écrouissage, } \\
\beta \quad: \text { paramètre de calage du modèle sans signification physique }
\end{aligned}
$$

Conformément à la plupart des modèles incluant un mécanisme plastique volumique, le potentiel correspondant à cette surface de charge est associé.

Dans la version utilisée sur le code de calcul CESAR-LCPC, ce modèle présente ainsi 7 paramètres :

. E, V : paramètres élastiques,

. $\beta \quad$ : paramètres de calage du modèle sans signification physique,

$. \varepsilon_{0}^{\mathrm{c}}, \varepsilon_{0}^{\mathrm{c}}$ : les déformations volumiquesélastique et plastique à la pression isotrope de référence $\mathrm{P} 0$

. $\phi_{\mathrm{cv}}, \phi_{\mathrm{p}}$ : angles de frottement à l'état critique et à l'état limite.

\subsection{Modèle de Drücker-Prager-Vermeer}

Comme on le verra plus loin, les simulations effectuées avec les modèles de MohrCoulomb et de Vermeer montrent les possibilités mais également les limites de ces

1. L'angle de dilatance $\psi_{\mathrm{m}}$ du modèle de Vermeer est fonction de l'angle de frottement mobilisé $\phi_{\mathrm{m}}$ et de l'angle de frottement à l'état critique $\phi_{\mathrm{cr}}$. Les angles $\psi$ (angle de dilatance du modèle de Mohr-Coulomb) et $\psi_{\mathrm{m}}$ ne représentent donc pas la même notion de dilatance. 
deux modèles de comportement. Il ressort notamment qu'un modèle à écrouissage s'impose mais qu'une formulation plus simple de la surface de charge pourrait éviter des calculs fastidieux voire des problèmes numériques. Aussi, nous avons programmé dans le code CESAR-LCPC un nouveau modèle plus robuste, conservant le principe de l'écrouissage du modèle de Vermeer, mais exploitant une surface de charge de cisaillement simplifiée, celle du modèle de Drücker-Prager.

$$
\begin{aligned}
& F_{c}\left(\sigma, \alpha_{c}\right)=\frac{q}{\sqrt{3}}-3 \cdot p \cdot \alpha_{c}(\chi) \\
& \alpha_{c}(\chi) \quad: \text { nouveau paramètre d'écrouissage, } \\
& \alpha_{c}(\chi)=\frac{H(\chi)}{3 \sqrt{3}}
\end{aligned}
$$

$\mathrm{H}(\chi) \quad$ : fonctionidentiqueà celle définie par Vermeer.

Le potentiel plastique de cisaillement garde la même forme que celle retenue dans la version simplifiée du modèle de Vermeer. Il présente ainsi la même forme conique que la surface de charge. L'angle de frottement à l'état caractéristique $\phi_{c v}$ est simplement adapté au nouvel angle de frottement interne, de manière à assurer un taux de dilatation identique à l'essai triaxial de compression.

Il convient toutefois de signaler que cette nouvelle surface de charge présente un inconvénient, celui de tolérer des contraintes de traction dans les milieux pulvérulents, pour un angle de frottement interne supérieur à $37^{\circ}$. Des précautions particulières ont ainsi été prises lors de l'application de ce modèle.

\section{Caractérisation mécanique du sol et du revêtement}

Les essais en centrifugeuse ont été réalisés sur le sable d'Hostun normalisé, sable préalablement testé à l'appareil triaxial [CHA 94] (Figure 2).

A l'état initial, la pression moyenne au niveau du tunnel est d'environ $80 \mathrm{kPa}$. Le sol est ensuite chargé en surface jusqu'à une pression de $1 \mathrm{MPa}$. Compte tenu de ces niveaux de contraintes, l'identification des paramètres a été effectuée sur trois essais triaxiaux à contrainte latérale constante de 100,300 et $600 \mathrm{kPa}$. Sur cette gamme de contraintes, et après l'état caractéristique $\left(d \varepsilon_{v}=0\right)$, il apparaît des taux de déformations volumiques $\left(\mathrm{d} \varepsilon_{\mathrm{v}}\right)$ par rapport aux déformations axiales $\left(\mathrm{d} \varepsilon_{\mathrm{axia}}\right)$, variables et donc incompatibles avec les hypothèses des deux modèles de comportement utilisés.

Il est rappelé que ces taux sont constants et égaux à :

$$
\begin{array}{ll}
\frac{\mathrm{d} \varepsilon_{\mathrm{v}}}{\mathrm{d} \varepsilon_{\text {axial }}}= & =\frac{-2 \cdot \sin (\psi)}{1-\sin (\psi)} \\
\frac{\mathrm{d} \varepsilon_{\mathrm{v}}}{\mathrm{d} \varepsilon_{\mathrm{axial}}} & =\frac{-6 \cdot \sin \left(\psi_{\mathrm{m}}\right)}{3-2 \cdot \sin \left(\psi_{\mathrm{m}}\right)} \quad \text { avec } \quad \sin \left(\psi_{\mathrm{m}}\right)=\frac{\sin \left(\phi_{\mathrm{p}}\right)-\sin \left(\phi_{\mathrm{cv}}\right)}{1-\sin \left(\phi_{\mathrm{p}}\right) \cdot \sin \left(\phi_{\mathrm{cv}}\right)} \quad \text { (Vermeer) }
\end{array}
$$


Mohr Coulomb
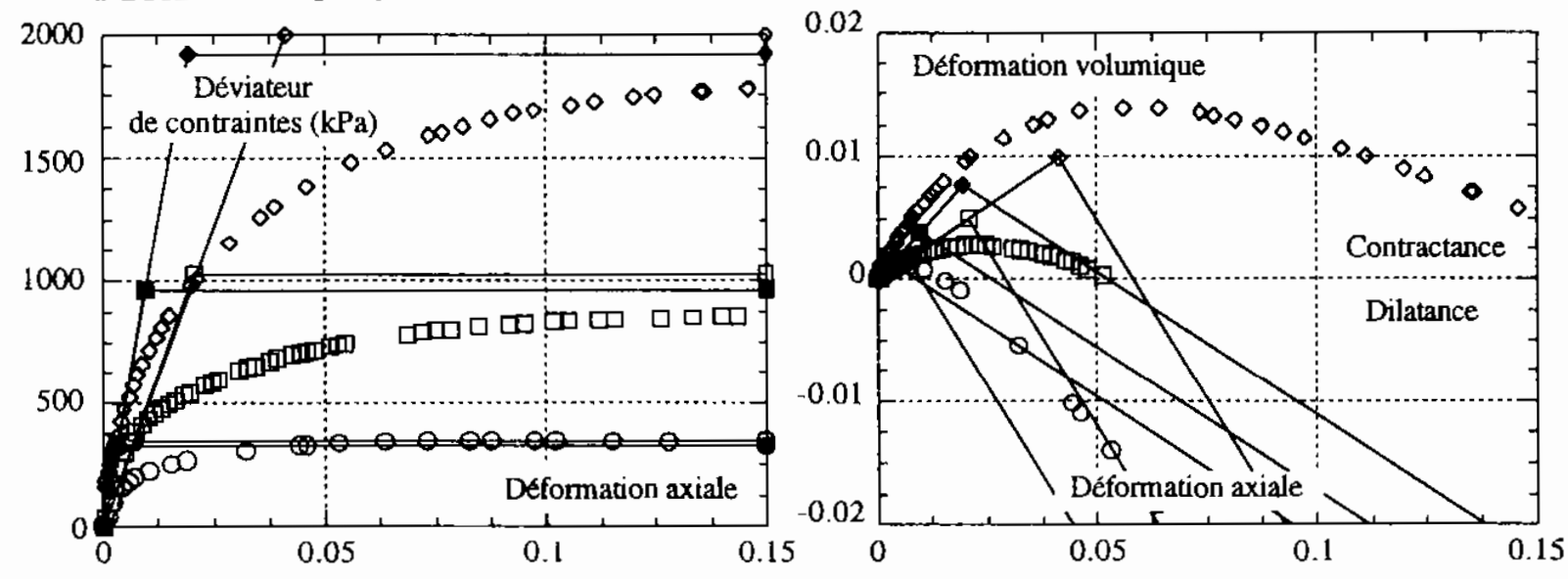

- Mesure $100 \mathrm{kPa}$

- Mesure $300 \mathrm{kPa}$

- Mesure $600 \mathrm{kPa}$

- Calcul - $100 \mathrm{kPa}$ : jeu $100 \mathrm{kPa}$

$\because-$ Calcul - $300 \mathrm{kPa}$ : jeu $100 \mathrm{kPa}$

- Calcul - $600 \mathrm{kPa}$ : jeu $100 \mathrm{kPa}$

$\rightarrow$ Calcul - $100 \mathrm{kPa}$ : jeu $600 \mathrm{kPa}$

Calcul - $300 \mathrm{kPa}$ : jeu $600 \mathrm{kPa}$

$\neg$ Calcul - $600 \mathrm{kPa}$ : jeu $600 \mathrm{kPa}$
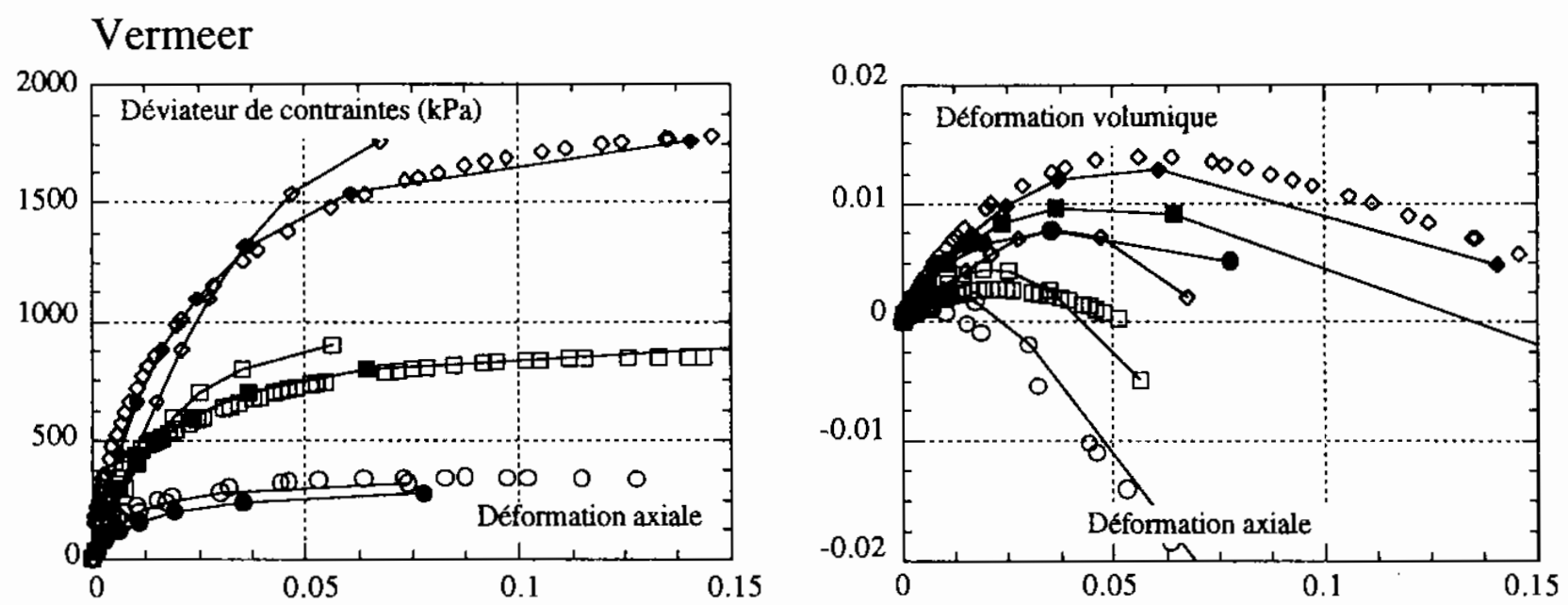

Figure 2. Essais triaxiaux - mesure et simulation. Triaxial tests - Hostun sand comparison of predicted and observed response

Pour cette raison, il a été convenu d'identifier deux jeux de paramètres par modèle, le jeu $100 \mathrm{kPa}$ et le jeu $600 \mathrm{kPa}$ correspondant aux deux niveaux extrêmes de contraintes. En ce qui concerne le modèle de Mohr-Coulomb, les paramètres $E$ et $v$ ont été établis sur la base de module et coefficient tangents initiaux, à défaut d'informations précises sur les valeurs moyennes des taux de contraintes à l'intérieur du massif lors du chargement. Les paramètres relatifs au modèle de Vermeer ont été déterminés selon la procédure décrite par Mestat [MES 90]. Nous rappelons que ce principe de détermination des paramètres repose sur une estimation des tangentes en certains points particuliers des courbes de compression triaxiale conventionnelle, et une identification avec les expressions théoriques de ces tangentes. 
Les jeux de paramètres, relatifs aux deux modèles, sont récapitulés dans les tableaux 1 et 2 . Les jeux du modèle de Vermeer obtenus suivant la procédure évoquée précédemment, ont été corrigés de manière à reproduire le plus fidèlement possible les résultats expérimentaux (Figure 2).

\begin{tabular}{|c|c|c|c|c|}
\hline & E MPa & $\nu$ & $\varphi$ & $\psi$ \\
\hline Jeu $-100 \mathrm{kPa}$ & 50 & 0,38 & $39,2^{\circ}$ & $13,0^{\circ}$ \\
\hline Jeu $-600 \mathrm{kPa}$ & 100 & 0,30 & $38,0^{\circ}$ & $6,0^{\circ}$ \\
\hline
\end{tabular}

Tableau 1. Sable d'Hostun-paramètres du modèle de Mohr-Coulomb. Hostun sand - sand properties - Mohr-Coulomb's model

\begin{tabular}{|c|c|c|c|c|c|c|c|}
\hline & E MPa & $v$ & $\beta$ & $\varepsilon_{0}{ }^{e}$ & $\varepsilon_{0}{ }^{\mathrm{c}}$ & $\phi_{\mathrm{cv}}$ & $\phi_{\mathbf{p}}$ \\
\hline Jeu $100 \mathrm{kPa}$ & 50 & 0,38 & 0,143 & 0,0074 & 0,0085 & $27,0^{\circ}$ & $39,2^{\circ}$ \\
\hline Jeu $600 \mathrm{kPa}$ & 100 & 0,30 & 0,070 & 0,0160 & 0,0160 & $33,0^{\circ}$ & $38,0^{\circ}$ \\
\hline
\end{tabular}

Tableau 2. Sable d'Hostun - paramètres du modèle de Vermeer. Hostun sand - sand properties - Vermeer's model

Le comportement du revêtement en Ertacétal est supposé élastique isotrope. Les paramètres, module d'Young et coefficient de Poisson, ont pour valeur respectivement $2900 \mathrm{MPa}$ et 0,25 . La fondation a une rigidité 1000 fois plus élevée que la rigidité maximale des éléments de sol.

\section{Discrétisation du massif, état initial et conditions aux limites}

Les simulations numériques ont été réalisées sur le code de calcul CESAR-LCPC en déformations planes. Les dimensions du modèle numérique respectent celles de la configuration prototype (Figure 1). Le maillage du demi espace est composé de 608 éléments à 6 nœuds (triangles) et à 8 nœuds (quadrilatères). Le revêtement du tunnel est représenté par 28 éléments (Figure 3).

Chacune des interfaces, fondation-sol et tunnel-sol, est modélisée par des éléments de contact à 6 nœuds. Conformément aux conditions expérimentales, les simulations de base ont été effectuées avec les conditions de contact suivantes :

- interface fondation-sol : contact lisse,

- interface tunnel-sol : contact parfaitement collé.

L'état initial est calculé en simulant une application progressive des forces volumiques dans le sol, de 1 à $50 \mathrm{~g}$, le tunnel étant libre de tout mouvement de translation et de déformation. Cette procédure respecte ainsi la préparation du massif de sol propre à l'essai en centrifugeuse. 


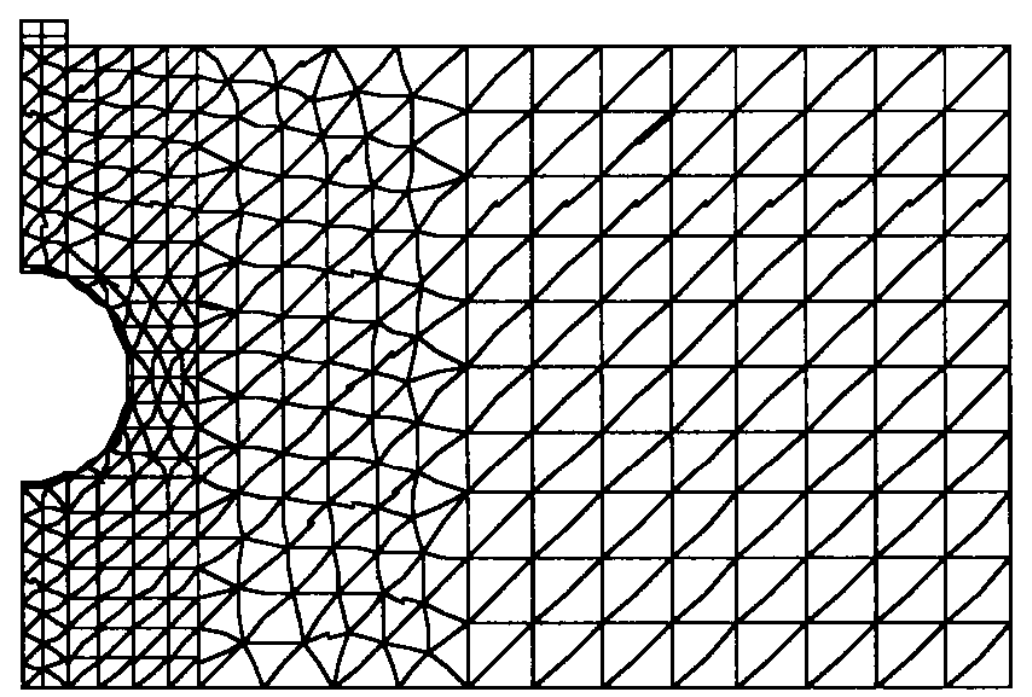

Figure 3. Maillage du massif de sol. Finite element mesh

\section{Incréments de chargement et critère de convergence}

Lors des simulations numériques, nous avons procédé à des chargements de la fondation superficielle par pas de $40 \mathrm{kPa}$. La plupart des calculs ont été réalisés avec un test de convergence portant sur un déséquilibre relatif maximum (Eps) de 0,01 et un maximum d'itérations $(\mathrm{Nmax})$ de 2000 . Ce critère de convergence a été régulièrement contrôlé par des études de sensibilité sur la taille des incréments de chargement et sur les paramètres Eps $\mathrm{r}_{\mathrm{r}}$ et $\mathrm{Nmax}$.

\section{Simulations numériques : modèles de Mohr-Coulomb, de Vermeer}

\subsection{Tassement de la fondation}

Les figures $4 \mathrm{a}$ et $4 \mathrm{~b}$ indiquent l'évolution du tassement de la fondation, mesuré et calculé, en fonction de la charge.

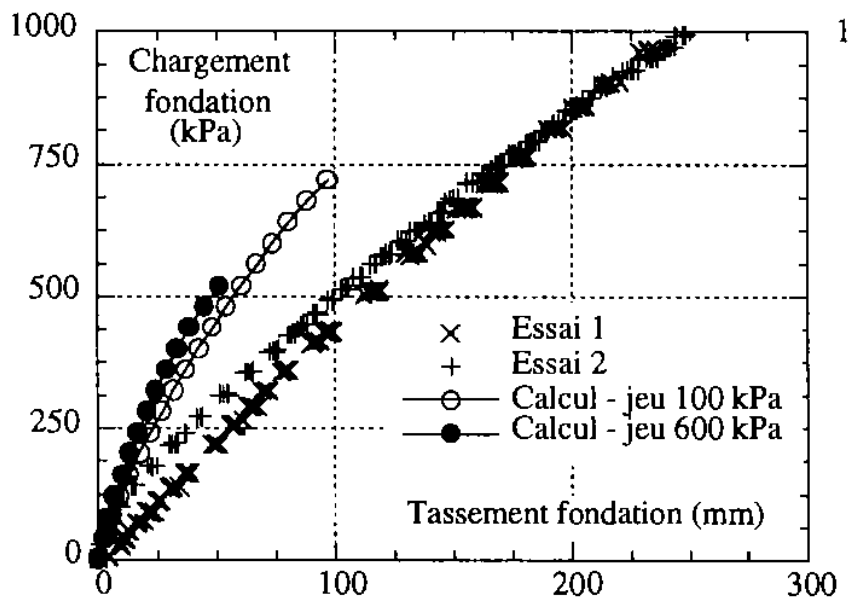

a . Modèle de Mohr-Coulomb

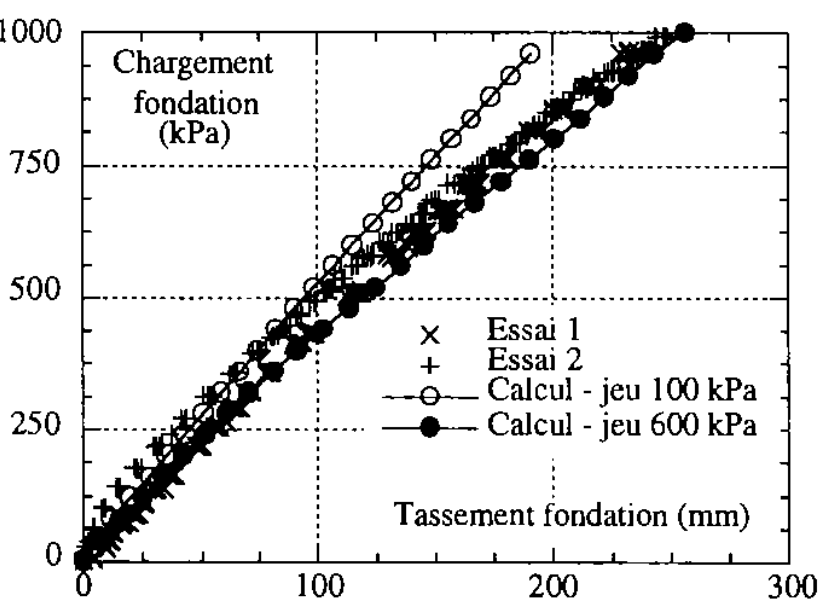

b. Modèle de Vermeer

Figure 4. Tassement de la fondation - mesures et simulations. Settlement curve observed and predicted load 
Nous y avons reporté les mesures des deux essais effectués sur la centrifugeuse ainsi que les résultats numériques correspondant aux deux jeux de paramètres des modèles de Mohr-Coulomb et de Vermeer.

\subsubsection{Modèle de Mohr-Coulomb}

On notera, tout d'abord, une sous-estimation des tassements calculés avec le modèle de Mohr-Coulomb, même si, lors de l'essai 2, le début du chargement laisse apparaître une rigidité plus élevée. Cet écart provient essentiellement du schéma élastoplastique parfait et du comportement élastique du sol évalué ici à partir du module d'Young tangent initial, en l'absence d'informations précises sur les taux de cisaillement. Il est clair que ce module ne peut convenir puisqu'on constate numériquement, a posteriori, des taux de déformations calculés atteignant des valeurs variant de $2 \%$ en périphérie de tunnel à $15 \%$ sous la fondation, pour un chargement de $600 \mathrm{kPa}$.

Par contre, la faible disparité entre les résultats des jeux $100 \mathrm{kPa}$ et $600 \mathrm{kPa}$ était moins prévisible. Pour un chargement de $500 \mathrm{kPa}$, l'accroissement de rigidité n'est que de $14 \%$ avec le jeu $600 \mathrm{kPa}$ alors que le module d'Young est deux fois plus élevé. Nous aurions pu compléter ces premiers résultats par une exploitation systématique de plusieurs jeux de paramètres satisfaisant à l'opération de calage sur les essais triaxiaux. Nous avons préféré déterminer la sensibilité des résultats vis-àvis de chacun des paramètres pris isolément. Il est toujours délicat, pour ce faire, d'établir un domaine de variation des valeurs de chaque paramètre. En l'absence d'une série d'essais plus conséquente sur ce matériau, nous avons adopté pour chacun de ces paramètres un domaine dont les bornes correspondent aux valeurs des jeux à 100 et $600 \mathrm{kPa}$, au risque d'exploiter des jeux non nécessairement réalistes.

Cette étude montre que le tassement de la fondation est régi non seulement par les paramètres élastiques $\mathrm{E}$ et $\mathrm{v}$, mais également par le paramètre de dilatance $\psi$ (Figure 5). Dans le cas présent, la rigidité plus élevée du jeu $600 \mathrm{kPa}$ est fortement compensée par un coefficient de Poisson et un angle de dilatance $\psi$ plus faibles (Tableau 3).

\begin{tabular}{|l|r|r|r|r|}
\hline & \multicolumn{1}{|c|}{$\begin{array}{c}\text { Jeu E } \\
\mathrm{E}^{*} 2\end{array}$} & $\begin{array}{c}\text { Jeu Nu } \\
\mathrm{v} / 1,27\end{array}$ & $\begin{array}{c}\text { Jeu Phi } \\
\varphi-1^{\circ} 2\end{array}$ & $\begin{array}{c}\text { Jeu Psi } \\
\psi-7^{\circ} 0\end{array}$ \\
\hline Incidence tassement & $-48 \%$ & $+21 \%$ & $+7 \%$ & $+22 \%$ \\
\hline Incidence charge ultime & $\cong 0$ & $\cong 0$ & $-17 \%$ & $-17 \%$ \\
\hline
\end{tabular}

Tableau 3. Tassement fondation - étude paramétrique - modèle Mohr-Coulomb. Foundation settlements - parametric study - Mohr-Coulomb's model

On retiendra donc que, pour ce type de configuration, deux jeux de paramètres, représentant chacun un comportement du matériau à deux niveaux de contraintes différents, fournissent des tassements de la fondation sensiblement équivalents. 


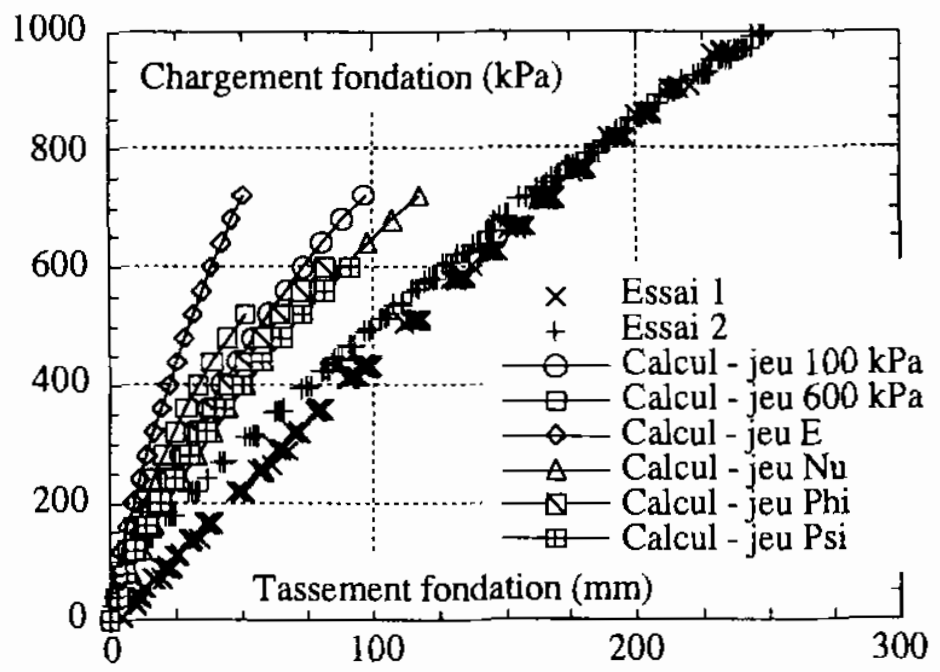

Jeu $\mathrm{E}:$ jeu $100 \mathrm{kPa} \quad$ avec $\mathrm{E} * 2 \quad \mathrm{E}$ du jeu $600 \mathrm{kPa}$ Jeu Nu : jeu $100 \mathrm{kPa}$ avec $\mathrm{v} / 1,27 \quad \mathrm{v}$ du jeu $600 \mathrm{kPa}$ Jeu Phi : jeu $100 \mathrm{kPa}$ avec $\varphi-1^{\circ} 2 \quad \varphi$ du jeu $600 \mathrm{kPa}$ jeu Psi : jeu $100 \mathrm{kPa}$ avec $\psi-7^{\circ} 0 \quad \psi$ du jeu $600 \mathrm{kPa}$

Figure 5. Tassement fondation - étude paramétrique - modèle Mohr-Coulomb. Foundation settlements - parametric study - Mohr-Coulomb's model

Par un calcul analytique en élasticité linéaire, et à partir des formules de Boussinesq, on peut montrer que le tassement d'un massif de sol homogène au centre d'une semelle est de la forme :

$$
\mathrm{h}=\alpha \cdot \frac{1-\mathrm{v}^{2}}{\mathrm{E}} \text { avec } \alpha: \text { fonction de la charge et de la forme de la semelle }
$$

Avec une telle relation, l'accroissement de rigidité devrait être $47 \%$ contre $14 \%$ dans le cas présent. Il s'avère donc que la plastification provoquée par le chargement de la fondation, la dilatance qui lui est associée, et le comportement du tunnel considéré en compression diamétrale comme incompressible, ne permettent plus d'exploiter une telle relation.

La propagation des zones plastiques en périphérie du tunnel au cours du chargement, provoque un tassement global de la fondation qui n'évolue pas de manière linéaire et qui est quelque peu différent du tassement observé expérimentalement. Par ailleurs, les simulations numériques font apparaître une non-convergence dans le processus itératif de résolution pour des valeurs du chargement comprises entre 500 et $700 \mathrm{kPa}$, valeurs qu'on assimilera ici aux charges limites et qui sont bien inférieures aux charges appliquées lors des essais. Cette non-convergence intervient lorsque la zone plastique débouchant en surface, recouvre la totalité du revêtement (Figure 6).

Le chargement numérique limite, qui n'est à associer à aucun phénomène physique constaté lors des essais, est directement lié aux angles de frottement interne et de 
dilatance. L'écart entre les charges limites obtenues avec les deux jeux de paramètres est de $28 \%$ : l'incidence de la variation de l'angle de frottement interne, $-1^{\circ} 2$, étant sensiblement la même que celle de la variation de l'angle de dilatance, $-7^{\circ} 0$ (Tableau 3). On remarquera que l'écart sur les charges limites, induit par la variation de l'angle de frottement interne, correspond à l'écart sur le coefficient $\mathrm{N} \gamma$ utilisé dans les formules analytiques de capacité portante d'une semelle en milieu pulvérulent, sans la présence du tunnel.

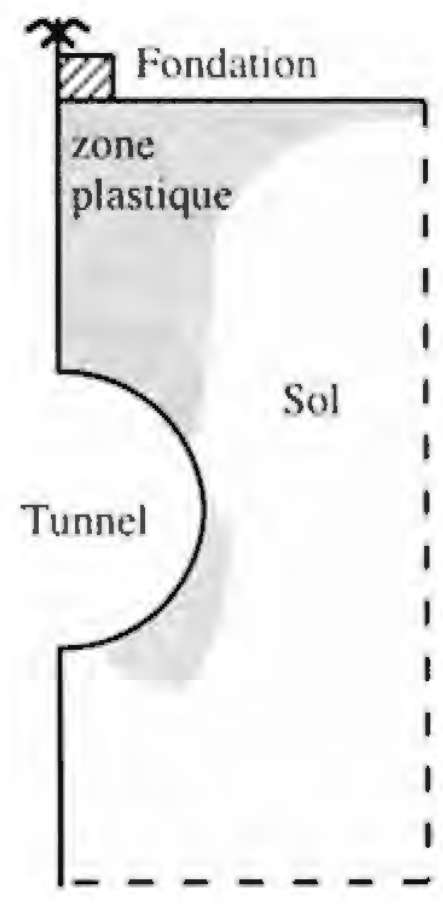

Figure 6. Zone déformation plastique $>2,0 \%$ précédent la rupture. Simulation Modele de Mohr-Coulomb. Plastic deformations (>2,0\%) before failure. Predicted load - Mohr-Coulomb's model

\subsubsection{Modèle de Vermeer}

La figure $4 \mathrm{~b}$ indique une bonne corrélation entre les tassements mesurés et ceux calculés avec le modèle de Vermeer. Son évolution est plus linéaire que celle calculée avec le modèle de Mohr-Coulomb. Elle est plus conforme aux résultats expérimentaux à variation linéaire, ceci malgré une plastification progressive du sol autour du tunnel et un comportement fortement écrouissable de ce matériau. Les tassements obtenus avec les deux jeux de paramètres encadrent les mesures des deux essais en centrifugeuse. Si l'écart entre les deux simulations numériques est encore réduit $(27 \%)$, il est plus élevé que celui mis en évidence avec le modèle de MohrCoulomb $(14 \%)$. Il faut signaler également que, contrairement à la simulation précédente, le massif de sol présente une plus grande souplesse avec le jeu $600 \mathrm{kPa}$, ceci malgré une raideur élastique plus élevée. Ce résultat est une conséquence de l'écrouissage qui donne au comportement plastique un rôle prépondérant. L'incidence de l'angle de dilatance est ainsi renforcée par rapport à celle observée avec modèle de Mohr-Coulomb. En l'occurrence, dans le cas présent, une dilatance 
plus faible se traduit par une souplesse que ne compense pas une raideur élastique deux fois plus élevée. La figure 7 et le tableau 4 précisent la sensibilité de chacun des paramètres, établie par une étude paramétrique semblable à la précédente.

\begin{tabular}{|l|c|c|c|c|c|c|c|}
\hline & Jeu E & Jeu Nu & Jeu Phicv & Jeu Béta & Jeu Phip & Jeu Eps0e & Jeu Eps0c \\
\hline $\begin{array}{l}\text { Incidence } \\
\text { tassement }\end{array}$ & $-29 \%$ & $+12 \%$ & $+36 \%$ & $+4 \%$ & $+3 \%$ & $+29 \%$ & $+14 \%$ \\
\hline
\end{tabular}

Tableau 4. Tassement de la fondation - étude paramétrique - modèle de Vermeer. Foundation settlement - predicted load - Vermeer's model

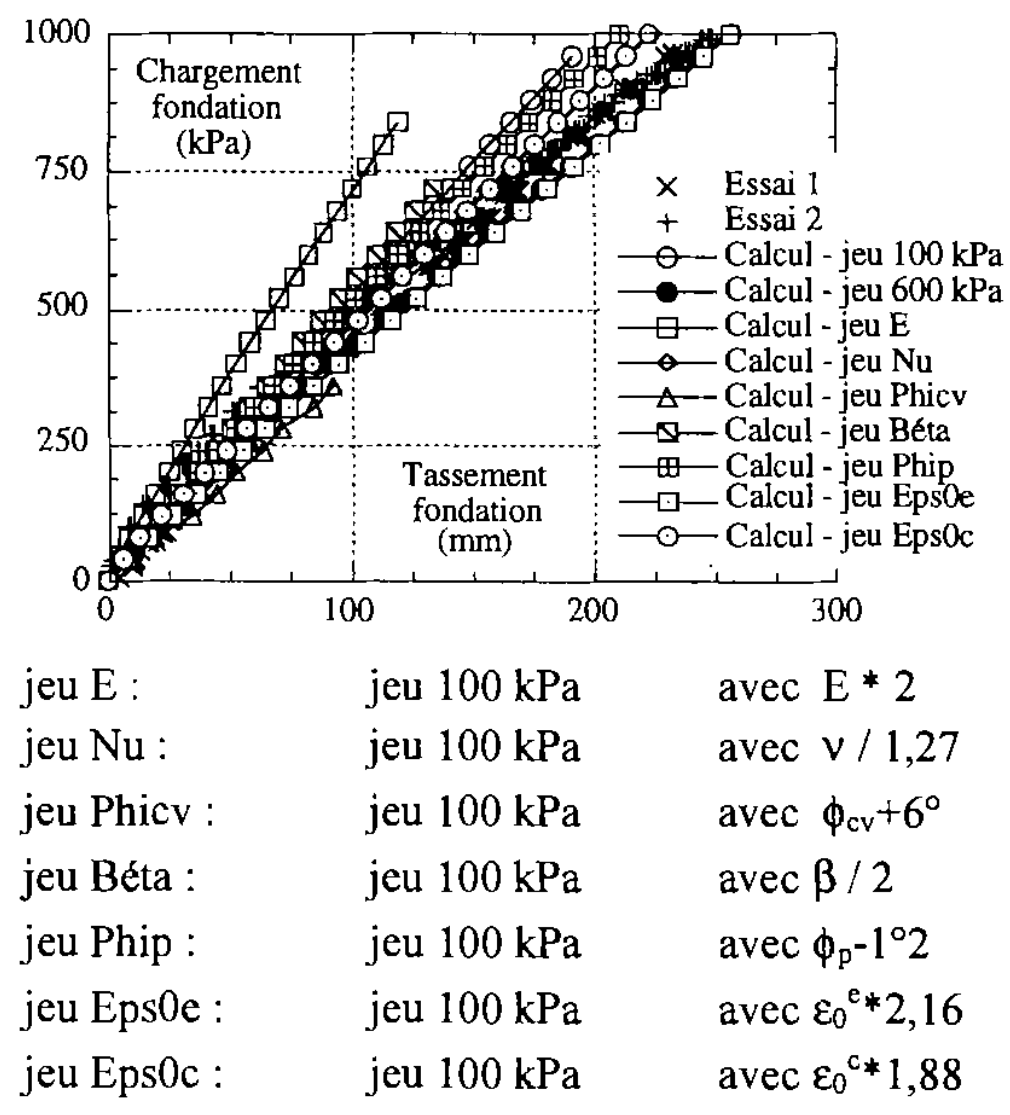

Figure 7. Tassement fondation - étude paramétrique - modèle de Vermeer. Foundation settlement - predicted load - Vermeer's model

Ce tableau met en évidence le rôle des paramètres plastiques intervenant dans le calcul des tassements pour un chargement de $500 \mathrm{kPa}$. Ainsi, on constate que l'incidence du module d'Young dans le modèle de Vermeer n'est plus que de $29 \%$ contre $48 \%$ dans le modèle de Mohr-Coulomb. L'effet du coefficient de Poisson est réduit à $12 \%$ contre $21 \%$ précédemment. Une variation de $6^{\circ}$ sur l'angle de dilatance, associée à un $\phi_{\mathrm{p}}$ de $39^{\circ} 2$, a pour conséquence par une perte de rigidité non compensée par la rigidité deux fois plus élevée du jeu $600 \mathrm{kPa}(+36 \%$ contre 
$-29 \%$ ). Dans la liste des paramètres exerçant une influence significative dans le calcul des tassements, il faut également citer les paramètres $\varepsilon_{0}{ }^{\mathrm{e}}$ et $\varepsilon_{0}{ }^{\mathrm{c}}$ intervenant dans les mécanismes plastiques de cisaillement et de consolidation.

Il apparaît ainsi que, dans le modèle de Vermeer, cinq paramètres jouent un rôle dans la réaction du sol sous la fondation. Par ailleurs, le poids de chacun de ces paramètres est globalement moins élevé que celui des paramètres exerçant une influence dans le modèle de Mohr-Coulomb. Ceci constitue un avantage dans la mesure où le résultat de la simulation n'est pas conditionnée par un nombre limité de paramètres dont l'identification est parfois délicate.

La difficulté dans l'identification des paramètres du modèle de Mohr-Coulomb réside principalement dans l'évaluation des paramètres élastiques, $E$ et $v$, dont les valeurs, constantes, doivent rendre compte du taux de contrainte moyenne au cours du chargement. L'identification avec le modèle de Vermeer ne présente pas cette difficulté puisqu'il dispose de paramètres d'écrouissage. Ces paramètres d'écrouissage permettent de formuler une relation contrainte-déformation fonction des chemins de contraintes suivis par le matériau. Il convient, cependant, de signaler que sur une série de simples essais triaxiaux en chargement, il n'est pas possible de déterminer tous les paramètres de manière unique. Ce constat apparaît clairement dans la démarche qui consiste à corriger le jeu de paramètres établi par identification analytique en des points particuliers de l'essai triaxial selon la procédure proposée par Mestat. Ce jeu doit, en effet, être adapté afin d'assurer la coïncidence sur l'ensemble des points de mesure, $q-\varepsilon_{\text {axisle }}$ et $\varepsilon_{v}-\varepsilon_{\text {axiale }}$. Cette procédure de calage est présentée ci-dessous. Elle a été justifiée par une étude de sensibilité semblable à la précédente, qui consiste à modifier tour à tour un des paramètres du jeu $100 \mathrm{kPa}$ par sa valeur du jeu $600 \mathrm{kPa}$ (Figure 8).
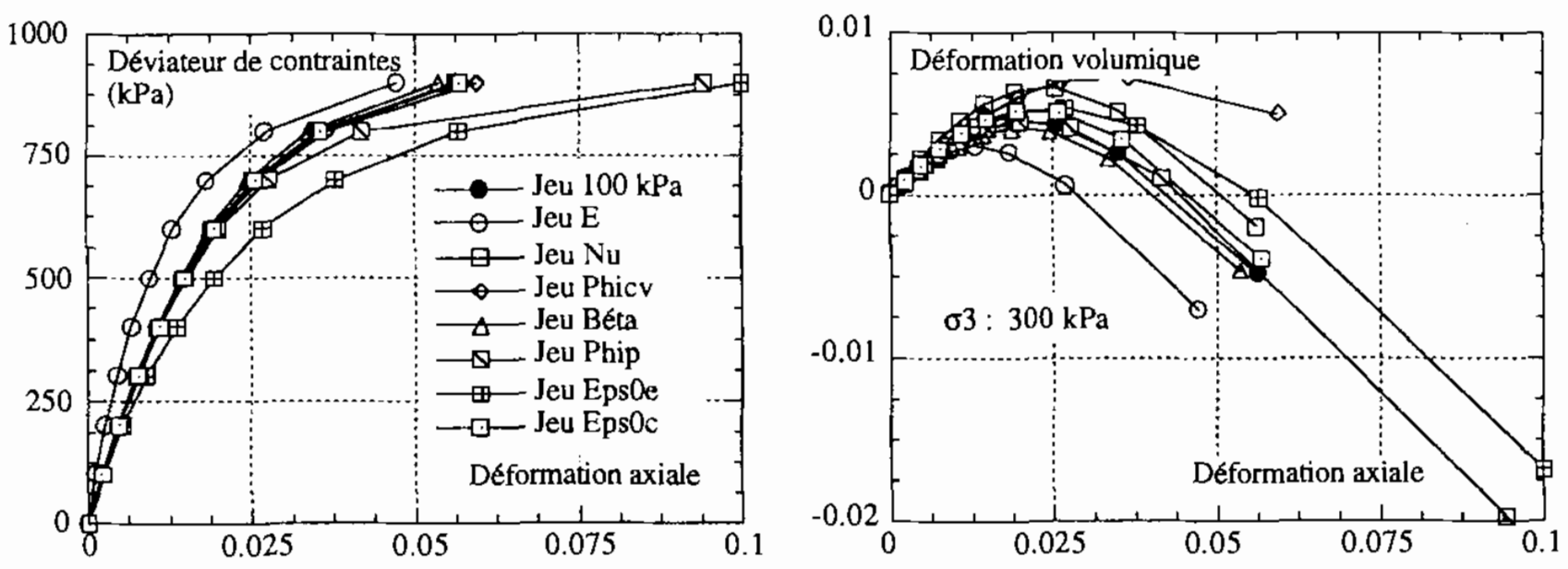

Figure 8. Essai triaxial - sable d'Hostun - étude de sensibilité aux paramètres modèle de Vermeer. Triaxial test - Hostun sand - parametric study - Vermeer's model 
Les paramètres $\mathrm{E}$ et $\phi_{p}$, conditionnant la pente à l'origine et l'asymptote à la rupture

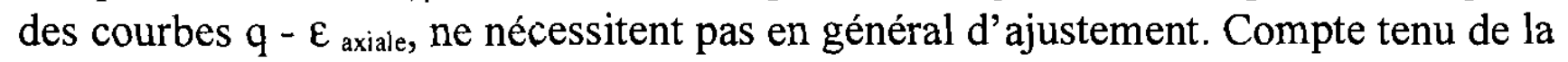
faible sensibilité des paramètres $v, \phi_{\mathrm{cv}}, \beta$ et $\varepsilon_{0}{ }^{\mathrm{c}}$, on retiendra essentiellement le paramètre $\varepsilon_{0}^{e}$ pour corriger leur courbure.

Les paramètres $v$ et $\phi_{\mathrm{cv}}$ définissent les courbes $\varepsilon_{\mathrm{v}}-\varepsilon_{\text {axiale }}$ à l'origine et à la rupture. Il ne reste donc à notre disposition que les paramètres $\beta$ et $\varepsilon_{0}^{c}$ pour corriger la position de l'état caractéristique en déformation volumique et en déformation axiale. Or, cet essai privilégiant le mécanisme de cisaillement ne permet pas de mettre en évidence le rôle de ces deux paramètres (Figure 8b). On regrettera en général l'absence de mesures à l'essai de compression isotrope, essai peu fréquent et délicat à réaliser en raison des faibles déformations qu'il engendre.

Pour l'ouvrage étudié, ce manque d'information ne constitue pas véritablement un handicap puisque la sensibilité à ces paramètres $\beta$ et $\varepsilon_{0}{ }^{\mathrm{c}}$ est relativement faible, respectivement $4 \%$ et $14 \%$.

Le modèle de Vermeer présente également l'avantage, par rapport au modèle de Mohr-Coulomb, de ne pas sous-estimer systématiquement la charge ultime. Avec les deux jeux de paramètres de base, le sol supporte "numériquement " la charge de $1 \mathrm{MPa}$ appliquée lors des essais en centrifugeuse. La prise en compte d'un écrouissage toujours positif, qui assure en toutes circonstances une rigidité non nulle, peut expliquer ce comportement. A noter, cependant, parmi les variantes, deux jeux de paramètres, le jeu E et le jeu Phicv, pour lesquels on observe une nonconvergence prématurée (Figure 7). Cette non-convergence est ici liée à des problèmes numériques. Elle n'a pas d'explication "mécanique » comme pour le modèle de Mohr-Coulomb où une zone plastifiée « parfaitement » au sens de MohrCoulomb recouvrait le tunnel.

\subsection{Variation diamétrale horizontale du tunnel}

La variation diamétrale du tunnel, mesurée et calculée, est reportée sur la figure 9.

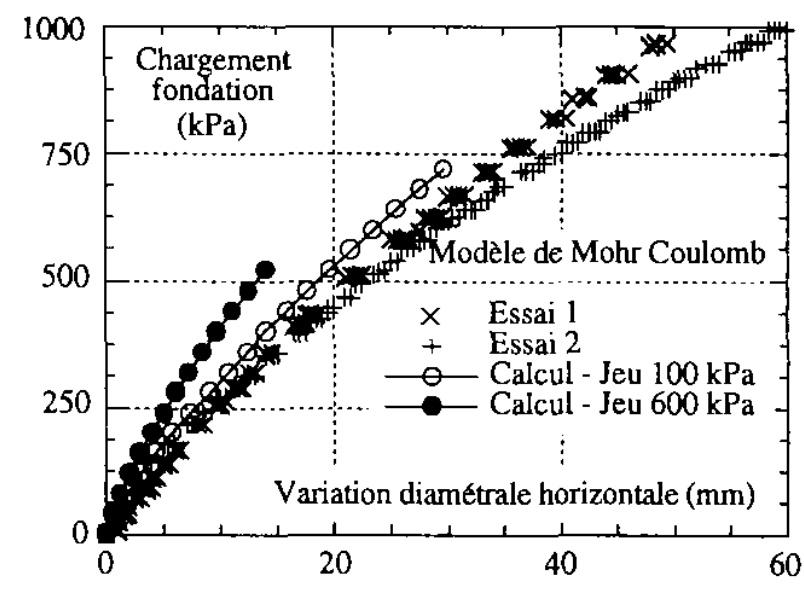

a . Modèle de Mohr-Coulomb

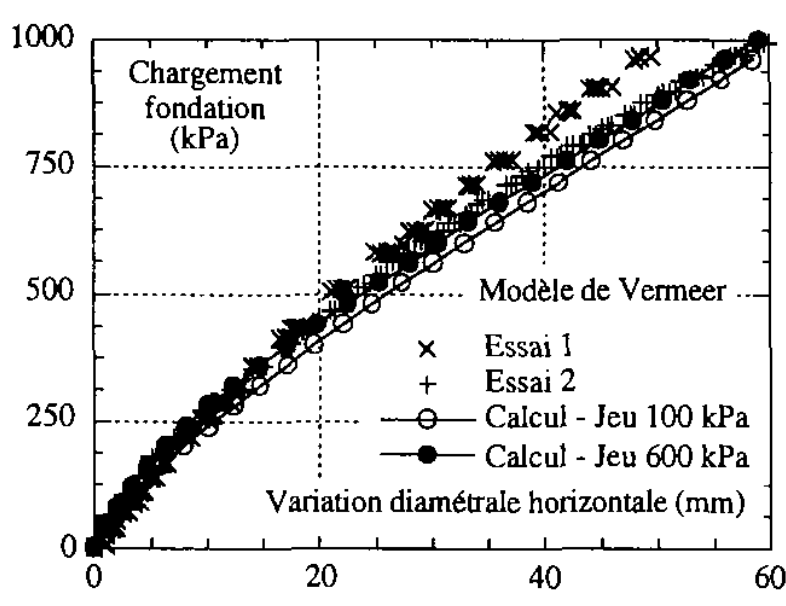

b. Modèle de Vermeer

Figure 9. Variation diamétrale horizontale du tunnel - mesures et simulations. Radial displacement of tunnel - observed and predicted displacement 


\subsubsection{Modèle de Mohr-Coulomb}

La réponse du tunnel en déformations, reproduite sur la figure $9 \mathrm{a}$, confirme la surestimation de la raideur du sol constatée lors de l'étude des tassements de la fondation. Cette tendance que l'on peut expliquer par le schéma élastoplastique parfait du modèle, est toutefois moins marquée au niveau du tunnel. Si on considère la valeur moyenne des résultats expérimentaux pour un chargement de $500 \mathrm{kPa}$, la surestimation de la raideur sur le tassement était de $82 \%$ avec le jeu $100 \mathrm{kPa}$ et $112 \%$ avec le jeu $600 \mathrm{kPa}$. Concernant les déformations du tunnel, celle-ci ne représente plus que $15 \%$ avec le jeu $100 \mathrm{kPa}$ et $61 \%$ avec le jeu $600 \mathrm{kPa}$.

Le fait que les résultats numériques sont plus conformes aux mesures dans cette partie du sol, résulte d'une raideur du matériau plus réaliste. Les modules d'Young tangents initiaux sont plus représentatifs des taux de contraintes moyennes et des taux de déformations déviatoires existant autour du tunnel lors du chargement. On perçoit ici les limites de ce modèle, du moins dans sa version à module d'Young constant.

Par ailleurs, la figure 9a fait état, en terme d'évolution de la déformation du tunnel, d'une réponse qualitativement très proche de la mesure. Son caractère non linéaire est reproduit correctement contrairement à ce qui était observé lors de l'étude des tassements. On constate, cependant, entre les résultats des jeux $100 \mathrm{kPa}$ et $600 \mathrm{kPa}$, un écart plus élevé en déformations diamétrales qu'en tassements, $28 \%$ contre $14 \%$ précédemment pour un chargement de $500 \mathrm{kPa}$. Afin de donner une explication à cet écart, il a été réalisé une étude paramétrique semblable à la précédente (Figure 10).

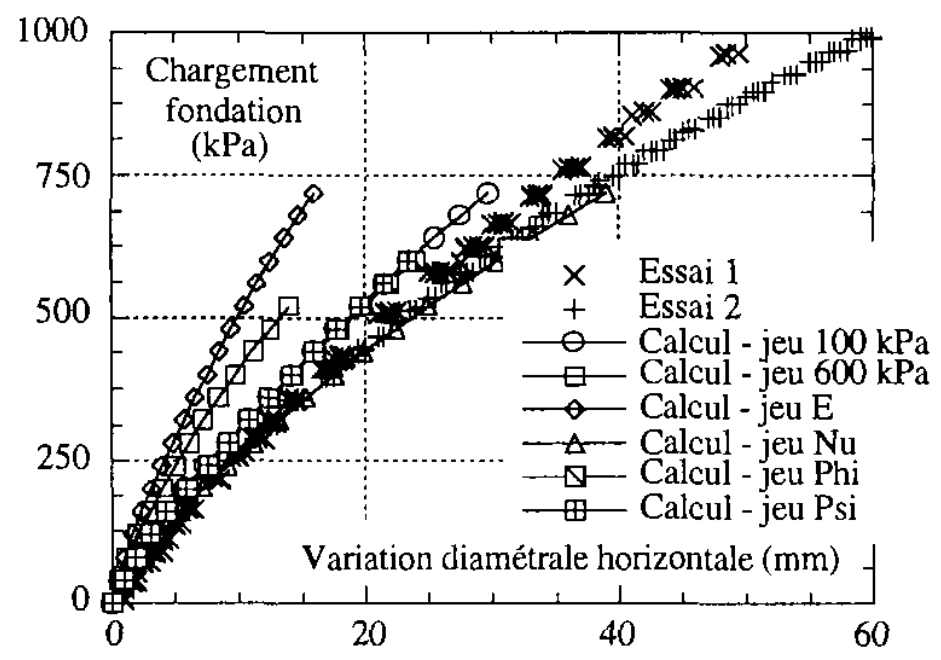

$\begin{array}{llll}\text { Simulation jeu E : } & \text { jeu } 100 \mathrm{kPa} & \text { avec } \mathrm{E}^{*} 2 & \mathrm{E} \mathrm{du} \mathrm{jeu} 2 \\ \text { Simulation jeu Nu : } & \text { jeu } 100 \mathrm{kPa} & \text { avec } v / 1,27 & v \text { du jeu } 2 \\ \text { Simulation jeu Phi : } & \text { jeu } 100 \mathrm{kPa} & \text { avec } \varphi-1^{\circ} 2 & \varphi \text { du jeu } \mathrm{n}^{\circ} 2 \\ \text { Simulation jeu Psi : } & \text { jeu } 100 \mathrm{kPa} & \text { avec } \psi-7^{\circ} 0 & \psi \text { du jeu } \mathrm{n}^{\circ} 2\end{array}$

Figure 10. Variation diamétrale horizontale du tunnel - étude paramétrique - MohrCoulomb. Radial displacement of tunnel - parametric study - Mohr-Coulomb 
On constate sur cette figure que seuls les paramètres élastiques, E et $v$, interviennent. Le comportement est essentiellement élastique dans la zone de sol adjacente au tunnel, zone qui subit l'effort de butée lié à l'ovalisation du tunnel. La déformation du tunnel n'est plus affectée par la variation de l'angle de dilatance.

Par ailleurs, le déplacement latéral provoqué par l'ovalisation, tel qu'il est défini dans la figure 11, est partiellement non contraint, notamment vers la surface. Il s'en suit une dépendance vis-à-vis de $v$ plus faible que dans le tassement de la fondation ( $19 \%$ contre $34 \%$ ), et une moindre compensation de l'effet de la variation du module d'Young sur cette déformée.

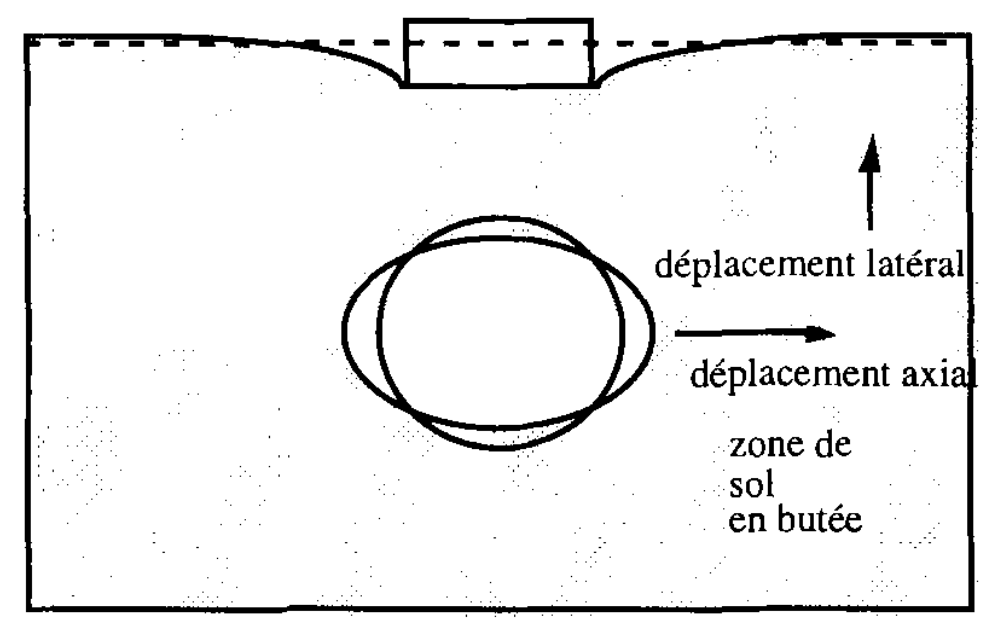

Figure 11. Mécanisme de butée dans la zone adjacente au tunnel, Passive thrust in the lateral zone

Ce raisonnement permet d'expliquer l'écart plus significatif entre les deux jeux de paramètres.

\subsubsection{Modèle de Vermeer}

Le modèle de Vermeer exploité, tant avec le jeu $100 \mathrm{kPa}$ qu'avec le jeu $600 \mathrm{kPa}$, permet de reproduire de manière satisfaisante la déformation horizontale du tunnel, notamment la déformation mesurée lors de l'essai 2 (Figure 9b). Les deux simulations rendent compte de la non-linéarité observée expérimentalement entre un chargement de $200 \mathrm{kPa}$ et $500 \mathrm{kPa}$, et du comportement linéaire au-delà de ce seuil. Le taux de déformation par rapport au chargement est globalement respecté. L'évolution des zones correspondant à une déformation plastique de cisaillement supérieure à $1 \%$, est représentée dans la figure 12. Elle montre que le comportement non linéaire est lié au développement de deux zones plastiques, l'une sous la semelle, l'autre située entre le rein et la clé de voûte du tunnel. Le comportement linéaire simulé au-delà de $500 \mathrm{kPa}$, débute lorsque ces deux zones se rejoignent. 


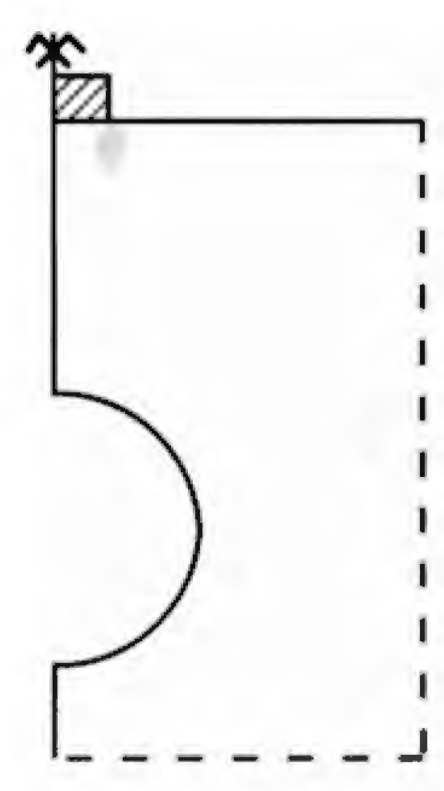

$\mathrm{a}: \mathrm{P}=200 \mathrm{kPa}$

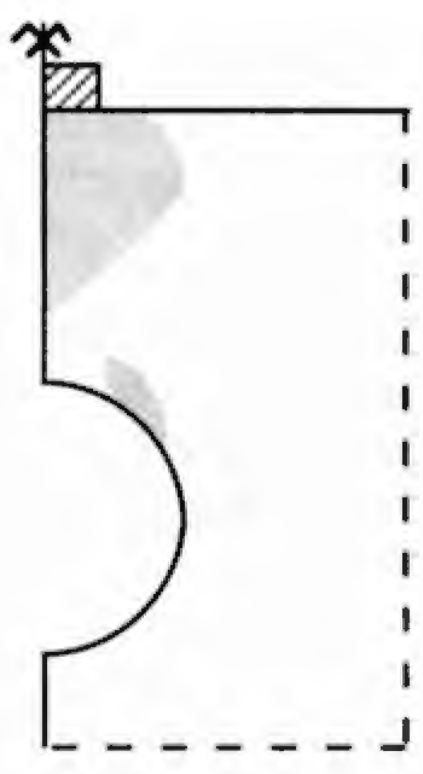

$\mathrm{b}: \mathrm{P}=400 \mathrm{kPa}$

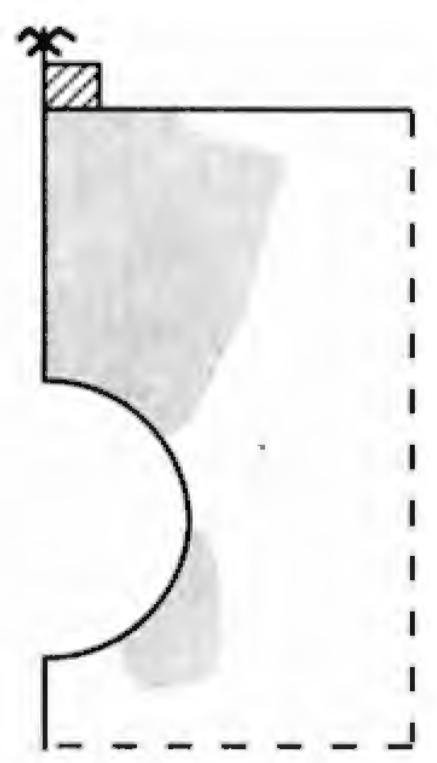

$\mathrm{c}: \mathrm{P}=1000 \mathrm{kPa}$

Figure 12. Zone à déformation plastique $>1.0 \%$ - modèle de Vermeer. Plastic Zones (>1.0\%) - Vermeer's model

Les simulations selon le modèle de Vermeer se distinguent de celles de MohrCoulomb par un comportement élastoplastique dans la zone adjacente au tunnel. L'étude paramétrique précise la dépendance de l'ovalisation du tunnel vis-à-vis des paramètres plastiques (Tableau 5, Figure 13).

Elle est globalement deux fois plus faible que celle mise en évidence lors de l'étude du tassement de la fondation, mais reste cependant significative. Elle compense, dans le jeux $600 \mathrm{kPa}$, l'augmentation de raideur par le module d'Young, conférant ainsi un comportement d'ensemble équivalent à celui proposé par le jeu $100 \mathrm{kPa}$. L'écart entre les deux simulations est de $8 \%$ à $500 \mathrm{kPa}$.

De cette analyse des déformations du massif de sol, on retiendra principalement que la dépendance de la raideur vis-à-vis de le pression moyenne et du taux de distorsion plastique (écrouissage) dans le modèle de Vermeer, permet une simulation, à la fois du tassement et de la déformation radiale du tunnel, conforme aux mesures. Le modèle de Mohr-Coulomb ne peut assurer cette concordance avec un seul jeu de paramètres. Concernant les charges limites, le modèle de Mohr-Coulomb les sousestime quelle que soit la variante étudiée. Cette charge correspond à un recouvrement du tunnel par les zones plastiques. Pour atteindre cette charge avec le modèle de Vermeer, il convient d'imposer des incréments de chargement suffisamment petits afin d'assurer la convergence du processus itératif de résolution. Pour deux jeux de paramètres, les jeux $E$ et Phicv, il n'a guère été possible d'atteindre la charge imposée lors des essais en centrifugeuse, quelle que soit la progression incrémentale (« raisonnable » en terme de temps de calcul) appliquée au chargement de la fondation. 


\begin{tabular}{|l|c|c|c|c|c|c|c|}
\hline & $\begin{array}{c}\text { Jeu } \\
\mathrm{E}\end{array}$ & $\begin{array}{c}\text { Jeu } \\
\mathrm{Nu}\end{array}$ & $\begin{array}{c}\text { Jeu } \\
\text { Phicv }\end{array}$ & $\begin{array}{c}\text { Jeu } \\
\text { Béta }\end{array}$ & $\begin{array}{c}\text { Jeu } \\
\text { Phip }\end{array}$ & $\begin{array}{c}\text { Jeu } \\
\text { Eps0e }\end{array}$ & $\begin{array}{c}\text { Jeu } \\
\text { Eps0c }\end{array}$ \\
\hline $\begin{array}{l}\text { Incidence } \\
\text { ovalisation }\end{array}$ & $-32 \%$ & $+15 \%$ & $+20 \%$ & $+0 \%$ & $+1 \%$ & $+14 \%$ & $+13 \%$ \\
\hline
\end{tabular}

Tableau 5. Variation diamétrale horizontale - étude paramétrique - modèle de Vermeer. Radial displacement of tunnel - parametric study - Vermeer's model

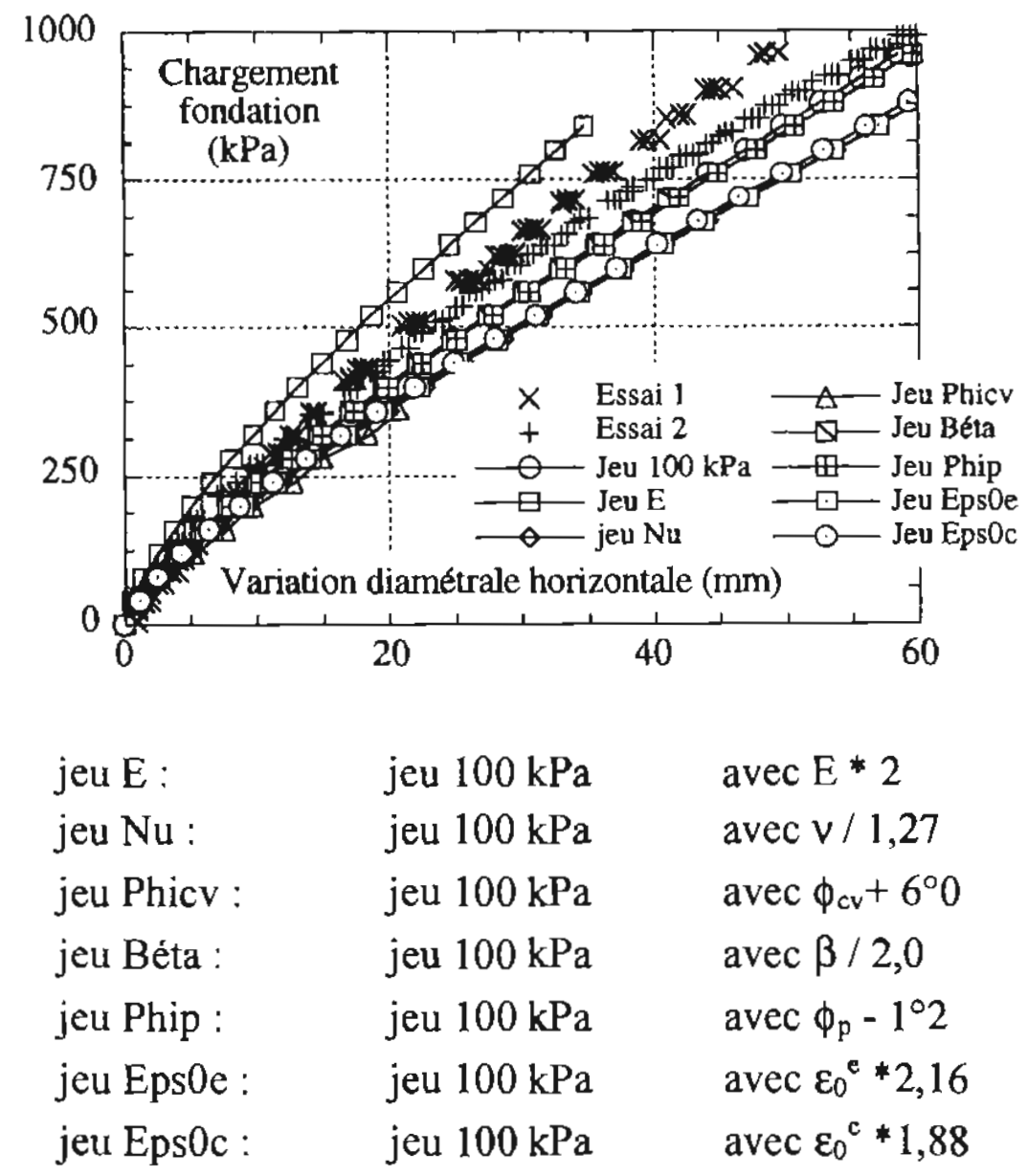

Figure 13. Variation diamétrale horizontale - étude paramétrique - Modèle de Vermeer. Radial displacement of tunnel - parametric study - Vermeer's model

\section{Modèle Drücker-Prager-Vermeer}

Dans le but de rechercher la cause de ces problèmes numériques constatés avec le modèle de Vermeer, nous avons été amenés à tester quelques aménagements sur la formulation de son écoulement plastique. Nous avons ainsi constaté que la prise en compte dans le modèle de Vermeer d'une surface de charge plus simple pouvait en partie éviter ces non-convergences. Le nouveau modèle proposé, considérant une surface de charge de Drücker-Prager et un écrouissage semblable à celui du modèle de Vermeer, a été testé avec le jeu $100 \mathrm{kPa}$ adapté à la nouvelle formulation. Les 
paramètres $\beta, \varepsilon_{0}{ }^{\mathrm{e}}$ et $\varepsilon_{0}{ }^{\mathrm{c}}$ ont ainsi été corrigés par calage avec les résultats des essais triaxiaux. Par ailleurs, sachant que cette nouvelle surface de rupture ne peut s'appliquer à tous les chemins de contrainte, deux surfaces ont été considérées : l'une correspondant à la rupture en compression, l'autre correspondant à la rupture en extension.

Les résultats en termes de déplacements sous la fondation et au rein du tunnel sont reportés sur les figures $14 \mathrm{a}$ et $14 \mathrm{~b}$.
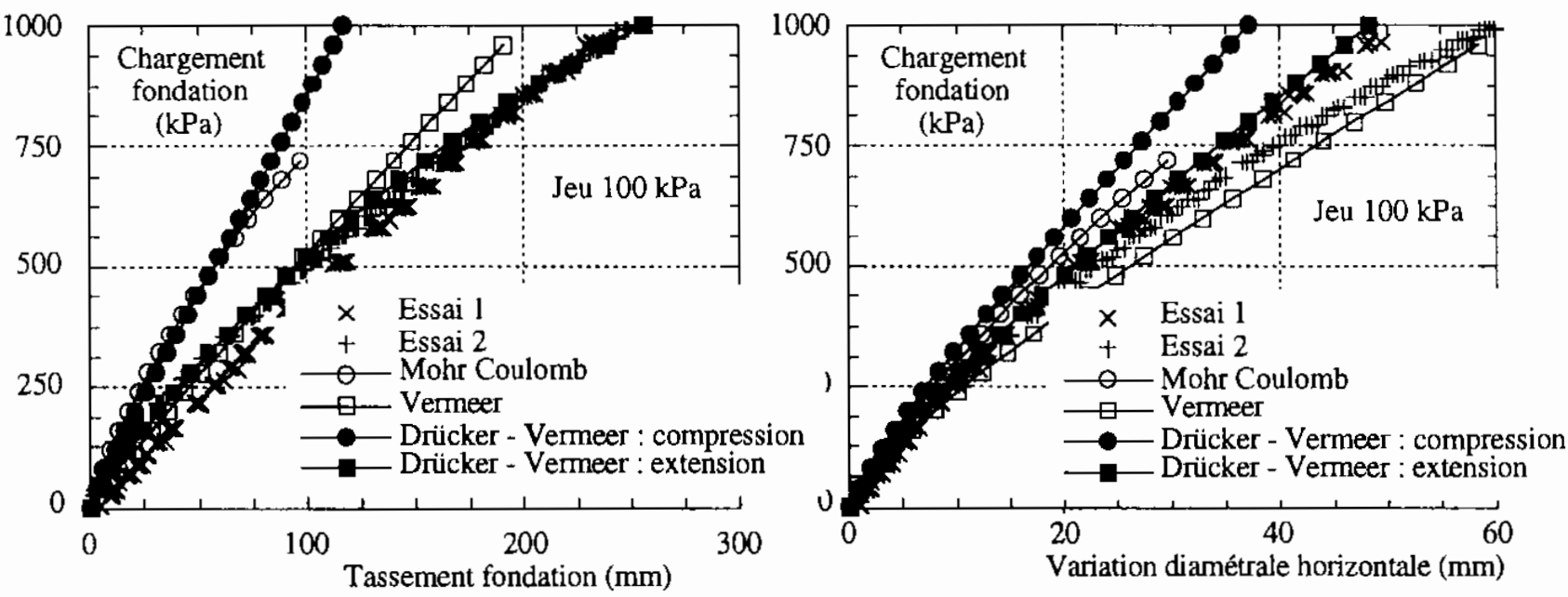

Figure 14. Tassement, variation diamétrale horizontale, modèle Drücker - Vermeer. Settlement and radial displacement of tunnel, Drücker - Vermeer Model

Ces résultats mettent en évidence une plus grande stabilité dans le processus de résolution, avec le modèle de Drücker - Prager - Vermeer. La convergence est assurée pour la charge maximale appliquée lors de l'essai en centrifugeuse, à savoir $1 \mathrm{MPa}$, quelle que soit la surface de rupture considérée. Ce test montre ainsi qu'une surface de charge exprimée en fonction du troisième invariant et donc plus réaliste peut être la cause de problèmes numériques.

Ce test avait pour objectif de rechercher la cause des problèmes numériques. On peut constater, par ailleurs, sur ce calcul que, si on considère la surface de charge établie par l'essai triaxial d'extension, la simulation des tassements de la fondation et de l'ovalisation du tunnel est aussi conforme aux mesures que celle fournie par le modèle de Vermeer. Cette surface de charge se justifie par le fait que, dans les zones plastifiées, la contrainte principale intermédiaire, qui agit ici sur le plan de déformation plane, tend au cours du chargement à se rapprocher de la contrainte principale majeure (Figures 15a et 15b).

Ce test montre ainsi que l'écriture d'un modèle doit non seulement respecter certains principes, dont ceux de la thermodynamique, et permettre de représenter les comportements sous sollicitations diverses, mais, de plus, elle doit proposer un système d'équations que le logiciel pourra résoudre avec suffisamment de précision 
et de façon stable : stabilité numérique particulièrement recherchée dans une application de type industriel. Ce test montre également qu'il peut être utile de comparer les résultats fournis par un modèle sophistiqué avec ceux fournis par un modèle plus simple assurant un meilleur conditionnement du système d'équations à résoudre. Cette confrontation exige toutefois que l'on maîtrise parfaitement le domaine de pertinence des modèles et le fonctionnement de l'ouvrage.

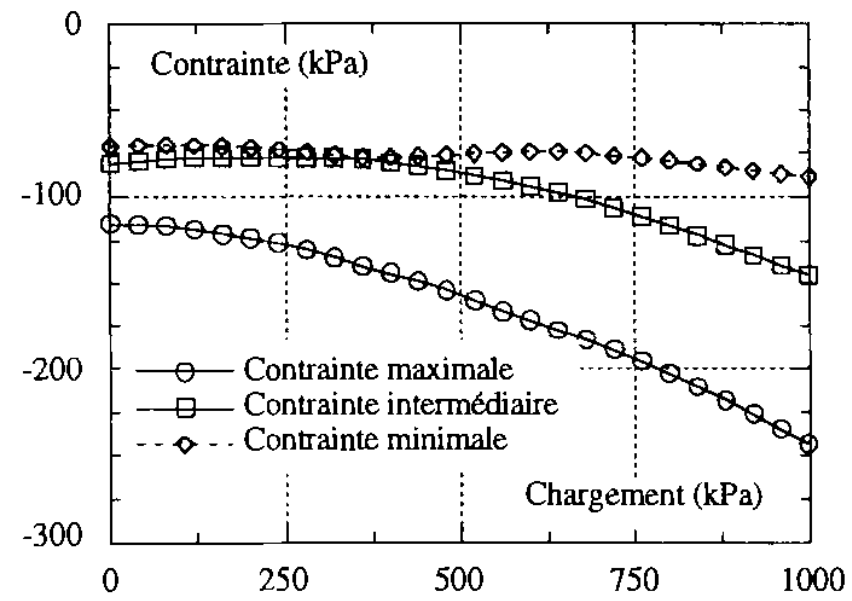

a. Point entre radier et rein

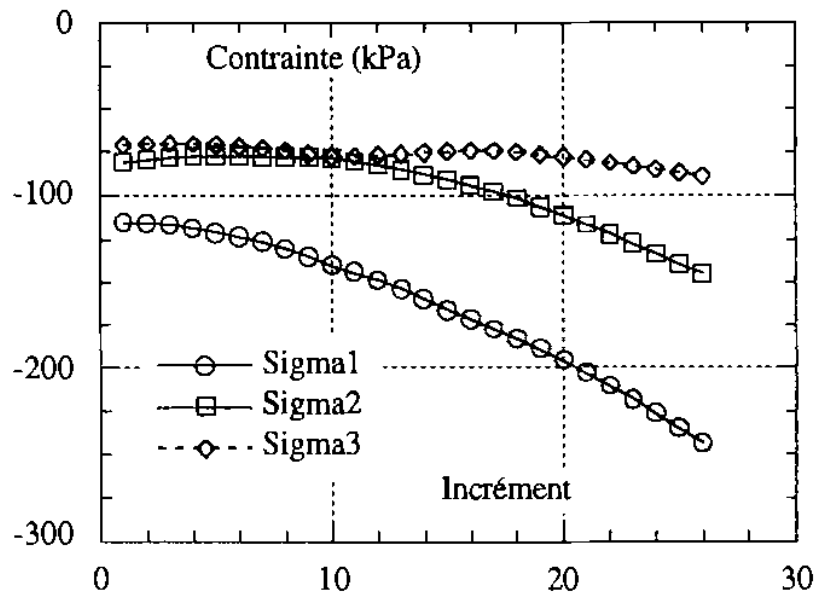

b. Point entre rein et clé de voûte

Figure 15. Evolution des contraintes principales, modèle Drücker - Prager Vermeer. Development of the principal stresses around the tunnel, Drücker Vermeer Model

\section{Conclusion}

L'étude que nous venons de présenter avait pour but de tester deux modèles de comportement, à degrés de sophistication différents, sur un ouvrage souterrain en service, ouvrage modélisé physiquement en centrifugeuse. De ce travail, on retiendra principalement que :

- les deux modèles utilisés ne permettent pas de représenter avec un jeu unique de paramètres le comportement du sable dans la gamme de contraintes à laquelle il est soumis. Cependant, les différentes simulations effectuées avec des jeux privilégiant chacun un niveau de contraintes particulier ne diffèrent que dans des proportions relativement faibles;

- le modèle de Mohr-Coulomb ne peut reproduire simultanément le comportement sous la fondation et au rein du tunnel avec le même jeu de paramètres. Il n'est pas non plus réaliste de proposer une valeur du module d'Young proportionnelle à la profondeur du fait que, dans le cas présent, la pression moyenne n'est pas répartie selon une fonction croissante de cette profondeur. De plus, ce modèle sous-estime fortement la charge limite ;

- le modèle de Vermeer, avec son écrouissage, permet de simuler correctement le comportement du sol aussi bien au niveau de la fondation qu'à proximité du tunnel. La sensibilité des paramètres, vis-à-vis du comportement de l'ouvrage, est 
globalement plus faible qu'avec le modèle de Mohr-Coulomb. Leur identification en est d'autant moins délicate que l'évaluation erronée d'un paramètre aura des conséquences limitées sur la réponse de l'ouvrage. On regrettera de n'avoir pas pu disposer dans cette étude d'essais de compression isotrope et d'essais de déchargement pour les essais triaxiaux et les essais en centrifugeuse. Ceux-ci nous auraient permis d'appréhender avec plus de précision le comportement volumique et le comportement élastique qui conditionnent la réponse du massif de sol ;

- le modèle de Vermeer peut dans certains cas conduire à un mauvais conditionnement du système d'équations à résoudre. Il a été montré que ce mauvais conditionnement, provoquant une non-convergence du processus de résolution, pouvait provenir de l'expression de la surface de charge et du potentiel plastique. Il conviendrait donc, lors de l'écriture des modèles, de proposer une formulation qui évite ces problèmes numériques rencontrés lors de la résolution. Les notions de robustesse et de stabilité qui dépendent non seulement de l'algorithme, mais également du système à résoudre, sont essentielles pour un code de calcul de type industriel et mérite d'être prise en compte dans l'écriture d'un modèle. Ce test montre également qu'il peut être utile de compléter certaines études en exploitant des modèles relativement simples qui assurent un meilleur conditionnement du système d'équations à résoudre. Ces simulations exigent toutefois que l'on maîtrise parfaitement le domaine de pertinence des modèles et le fonctionnement de l'ouvrage.

Le travail réalisé ici représente une des opérations permettant de valider un logiciel dans une procédure d'assurance qualité [GIB 94]. Dans le cas présent, avec le modèle de Vermeer et les deux calculs de base (jeux 100 et $600 \mathrm{kPa}$ ), nous pouvons considérer que ce critère est satisfait au sens où la Commission Internationale des Grands Barrages (CIGB) l'entend puisque la différence relative entre les valeurs numériques et expérimentales est de l'ordre de 5 à $20 \%$. Quant à estimer que ce calcul est un "bon calcul», seule une confrontation de ces résultats avec de nouvelles simulations intégrant des algorithmes de résolution et des modèles rhéologiques différents, peut apporter un élément de réponse. Cette opération constitue un autre critère dans la validation des logiciels de calcul [GIB 94].

\section{Remerciements}

Les expérimentations rapportées dans ce document ont été réalisées sur la centrifugeuse du LCPC - Centre de Nantes. Les auteurs remercient le personnel de ce centre et $\mathrm{P}$. Chambon (Ecole Centrale de Nantes) pour l'accès aux mesures expérimentales présentées ici. 


\section{Bibliographie}

[CHA 94] ChAmbon P., Souterrains et forages en site urbain, rapport interne GEO, Ecole Centrale de Nantes, Laboratoire de Génie Civil de Nantes-Saint Nazaire, 1994.

[CIB 94] «Computer software for dams - validation, comments and proposal », bulletin de la Commission Internationale des Grands Barrages, CIBG-ICOLD, 1994.

[DAR 95] DaRve F., Hicher P.Y., ReYNouARD J.M., Mécanique des Géomatériaux, Editions Hermès, Paris, 1995.

[MES 90] Mestat PH., Validation numérique du progiciel Cesar-LCPC pour les lois de comportement élastiques non linéaires plastiques, rapport interne LCPC, 1990.

[TAD 85] TADJBAKHSH S:, FRANK R., Etude par la méthode des éléments finis du comportement élastoplastique de sols dilatants : application aux pieux sous charge axiale, rapport de recherche LCPC, 1985.

[VER 82] VERMEER P.A., "A five-constant model unifying well-established concepts ", Results of the International Workshop on Constitutive Relations for Soils, Grenoble, 1982. 